\title{
Thermal Diffusion (Soret Effect) on an Unsteady MHD Mixed Convective Heat and Mass Transfer Flow through Vertical Porous Medium with Chemical Reaction
}

\author{
Sujan Sinha ${ }^{1}$ and Maushumi Mahanta ${ }^{2}$ \\ ${ }^{1}$ Assistant Professor, Department of Mathematics, Assam Down Town University, \\ Guwahati-26, Assam \\ ${ }^{2}$ Assistant Professor, Department of Mathematics, Saraighat College, \\ Changsari-101, Assam \\ E-mail: ${ }^{1}$ mathssujangu@gmail.com, ${ }^{2}$ maths.mmahanta@gmail.com
}

\begin{abstract}
A parametric study to investigate the effect of thermal diffusion (Soret effect) on an MHD mixed convective heat and mass transfer flow of an incompressible viscous electrically conducting fluid past a vertical porous plate. The magnetic Reynolds number is assumed to be so small that the induced magnetic field can be neglected as compared with the applied magnetic field. The resultant set of the non-dimensional governing equations are solved analytically by adopting perturbation technique. The profiles of the velocity, temperature, concentration, skin friction, Nusselt number and Sherwood number at the plate are demonstrated graphically for various values of the parameters involved in the problem and the results are physically interpreted. It is found in our discussion that thermal diffusion effect raises the fluid flow.
\end{abstract}

Keywords: Thermal-Diffusion, MHD, Dimensional, Perturbation Technique, Heat and Mass Transfer

\section{INTRODUCTION}

MHD is the science of movement in which all the characteristics of fluid with the magnetic benefits under the conduction of electric current. There are lots of applications of MHD principles in Engineering, Plasma Physics, in area of Biotechnology and Bio medical science. The effects of MHD in various heat and mass transfer problems with mixed convection are applied by several authors such as Elbashbeshy (2003), Singh et al. (2000) and Ahmed (2010).

Soret effect which is also known as thermal-diffusion effect concerns with the methods of separating heavier gas molecules from lighter ones by maintaining temperature gradient over a volume of a gas containing particles of different masses. The mass flux created by temperature gradient is termed as Soret or thermal -diffusion effect. In view of the importance of Soret effect, several authors have carried out their research works to investigate the problems related to thermal-diffusion effect. Some of them are Anghel et al. (2000), Postenlnicu (2004), Alam et al. (2004) and Ahmed (2010).

Several investigators have studied the impact of reaction in several convective heat and mass transfer flows of whom Apelblat (1982) and Anderson et.al (1994) are price mentioning. Chambre and Young (1958) have conferred a primary order reaction within the neighbourhood of a horizontal plate. Muthucumaraswamy (2002) conferred heat and mass transfer effects on an endlessly moving isothermal surface with uniform suction by taking in to account the homogenous reaction of 1st order.

The main aim of present study is to investigate the effect of thermal-diffusion (Soret effect) on a mixed convective heat and mass transfer flow through vertical porous plate with MHD. The work is an extension to the work done by Allan and Derbery (2018). 


\section{MATHEMATICAL FORMULATION OF THE PROBLEM}

In the present study, an MHD conducting heat and mass transfer flow of a viscous incompressible electrically conducting fluid past an infinite vertical porous plate with constant suction is considered as well as a uniform transverse magnetic field. This investigation is considered with the following assumptions:

- The polarization effects are assumed to be negligible and so electric field is also negligible.

- The suction velocity is constant.

- The variations of all fluid properties other than the variation of fluid density are completely ignored.

- When the plates are infinite, all the physical variables are functions of $\mathrm{y}^{\prime}$ and $\mathrm{t}^{\prime}$ only.

- It is assumed that the variation of expansion coefficient is negligibly small and assumed pressure and influence of the pressure on the density is negligible.

- The free stream oscillates about a steady mean.

Here, it is assumed $U^{\prime}(t)=U_{0}\left(1+\varepsilon e^{i \omega t}\right)$, where $U_{0}$ is the mean stream velocity, $\varepsilon$ is the amplitude of the free stream variation and $\mathrm{U}^{\prime}$ is velocity along the plate.

We introduce a co-ordinate system, where X-axis vertically upwards along the plate, Y-axis remains perpendicular to the plate and directed to the fluid region and Z-axis remains along the width of the plate as shown in Figure 1

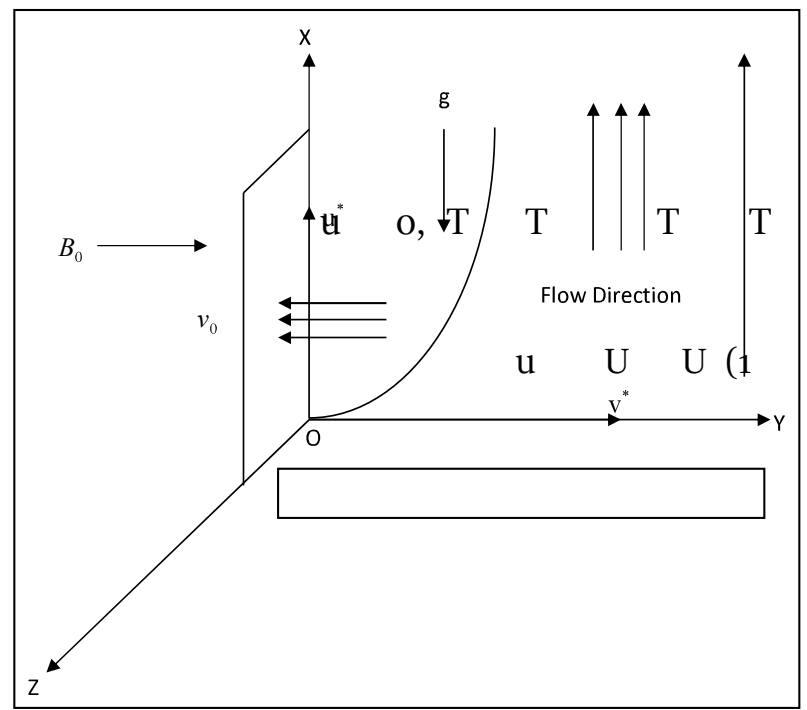

Fig. 1: Physical Model of the Problem
Let the components of velocity along the X-axis be $\mathrm{u}^{\prime}$ and components of velocity along the Y-axis be $\mathrm{V}^{\prime}$. So $\mathrm{u}^{\prime}$ is chosen in the upward direction along the plate and $\mathrm{V}^{\prime}$ are chosen normal to the plate.

Under the given assumptions, the equations that describe the physical situation are given by:

\section{Equation of Continuity:}

$$
\frac{\partial \mathrm{v}^{\prime}}{\partial \mathrm{y}^{\prime}}=0 \Rightarrow \mathrm{v}^{\prime}=-\mathrm{v}_{0}
$$

\section{Momentum Equation:}

$$
\begin{aligned}
\frac{\partial \mathrm{u}^{\prime}}{\partial \mathrm{t}^{\prime}}+\mathrm{v}^{\prime} \frac{\partial \mathrm{u}^{\prime}}{\partial \mathrm{y}^{\prime}}=-\frac{1}{\mathrm{p}} \frac{\partial \mathrm{p}^{\prime}}{\partial \mathrm{x}^{\prime}}+\mathrm{v} \frac{\partial^{2} \mathrm{u}}{\partial \mathrm{y}^{\prime 2}}-\frac{\sigma \beta_{o}^{2}}{\rho} \mathrm{u}^{\prime} \quad \mathrm{g} \quad \mathrm{T} \quad \mathrm{T} \quad \mathrm{g} \quad \mathrm{c} \quad \mathrm{c} \\
\mathrm{u}+\mathrm{g} \beta\left(\mathrm{T}^{\prime}-\mathrm{T}_{\infty}\right)+\mathrm{g} \beta_{\mathrm{c}}\left(\mathrm{c}^{\prime}-\mathrm{c}_{\infty}^{\prime}\right)-\mathrm{v} \frac{\mathrm{u}^{\prime}}{\mathrm{k}^{\prime}}
\end{aligned}
$$

\section{Energy Equation:}

$$
\frac{\partial \mathrm{T}^{\prime}}{\partial \mathrm{t}^{\prime}}+v^{\prime} \frac{\partial \mathrm{T}^{\prime}}{\partial \mathrm{y}^{\prime}}=\frac{\mathrm{k}}{\rho \mathrm{c}_{\mathrm{p}}} \frac{\partial^{2} \mathrm{~T}^{\prime}}{\partial \mathrm{y}^{\prime 2}}-\frac{1}{\rho \mathrm{c}_{\mathrm{p}}} \frac{\partial \mathrm{q}_{\mathrm{r}}}{\partial \mathrm{y}^{\prime}}
$$

\section{Concentration Equation:}

$$
\frac{\partial \mathrm{c}^{\prime}}{\partial \mathrm{t}^{\prime}}+v^{\prime} \frac{\partial \mathrm{c}^{\prime}}{\partial \mathrm{y}^{\prime}}=\mathrm{D} \frac{\partial^{2} \mathrm{c}^{\prime}}{\partial \mathrm{y}^{\prime 2}}-\gamma\left(\mathrm{c}^{\prime}-\mathrm{c}_{\infty}^{\prime}\right)+\mathrm{D}_{\mathrm{T}} \frac{\partial^{2} \mathrm{~T}^{\prime}}{\partial \mathrm{y}^{\prime 2}}
$$

The boundary conditions for the velocity, temperature and concentration fields are:

$$
\begin{aligned}
\mathrm{u}^{\prime} & =\mathrm{o}, \mathrm{T}^{\prime}=\mathrm{T}_{\mathrm{w}}^{\prime}+\varepsilon\left(\mathrm{T}_{\mathrm{w}}^{\prime}-\mathrm{T}_{\infty}^{\prime}\right) \mathrm{e}^{\mathrm{i} \omega \mathrm{t}}, \mathrm{c} \quad \mathrm{c} \\
\mathrm{e}, \mathrm{c}^{\prime} & =\mathrm{c}_{\mathrm{w}}^{\prime}+\varepsilon\left(\mathrm{c}_{\mathrm{w}}^{\prime}-\mathrm{c}_{\infty}^{\prime}\right) \mathrm{e}^{\mathrm{i} \omega \mathrm{t}} \text { at } \mathrm{y}^{\prime}=\mathrm{o} \\
\mathrm{u}^{\prime} & \rightarrow \mathrm{U}^{\prime}=\mathrm{U}_{\mathrm{o}}\left(1+\varepsilon \mathrm{e}^{\mathrm{i} \omega \mathrm{t}}\right), \mathrm{T} \quad \mathrm{T}, \mathrm{c} \quad \mathrm{c} \text { at } \mathrm{y} \\
\mathrm{e} \quad \mathrm{T}^{\prime} & \rightarrow \mathrm{T}_{\infty}^{\prime}, \mathrm{c}^{\prime} \rightarrow \mathrm{c}_{\infty}^{\prime} \text { at } \mathrm{y}^{\prime} \rightarrow \infty
\end{aligned}
$$

Radiative heat flux term is given by

$$
\frac{\partial \mathrm{q}_{\mathrm{r}}}{\partial \mathrm{y}^{\prime}}=4 \alpha^{2}\left(\mathrm{~T}_{\infty}^{\prime}-\mathrm{T}^{\prime}\right)
$$

Outside the boundary layer we consider

$$
-\frac{1}{\rho} \frac{\partial \rho^{\prime}}{\partial \mathrm{x}^{\prime}}=\frac{{\mathrm{dU^{ \prime }}}^{\prime}}{\mathrm{dt}^{\prime}}+\frac{v}{\mathrm{~K}^{\prime}} \mathrm{U}^{\prime}+\frac{\sigma}{\rho} \mathrm{B}_{0}^{2} \mathrm{U}^{\prime}
$$


We now consider the following dimensionless variables as follows:

$$
\begin{aligned}
& \mathrm{u}=\frac{\mathrm{u}^{\prime}}{\mathrm{U}_{0}}, \mathrm{y}=\frac{\mathrm{V}_{0} \mathrm{y}^{\prime}}{v}, \omega=\frac{v \omega^{\prime}}{\mathrm{v}_{0}^{2}}, \quad \mathrm{U}=\frac{\mathrm{U}^{\prime}}{\mathrm{U}_{0}} \\
& \mathrm{t}=\frac{\mathrm{t}^{\prime} \mathrm{V}_{0}^{2}}{v}, \theta=\frac{\mathrm{T}^{\prime}-\mathrm{T}_{\infty}^{\prime}}{\mathrm{T}_{\mathrm{w}}^{\prime}-\mathrm{T}_{\infty}^{\prime}}, \phi=\frac{\mathrm{C}^{\prime}-\mathrm{C}_{\infty}^{\prime}}{\mathrm{C}_{\mathrm{w}}^{\prime}-\mathrm{C}_{\infty}^{\prime}} \\
& \mathrm{N}=\frac{4 \alpha^{2} v}{\rho \mathrm{c}_{\mathrm{p}} \mathrm{v}_{0}^{2}} \mathrm{~K}=\frac{\mathrm{K}^{\prime} \mathrm{V}_{0}^{2}}{v^{2}}, \operatorname{Pr}=\frac{\rho v \mathrm{c}_{\mathrm{p}}}{\mathrm{k}} \\
& \mathrm{M}=\frac{\sigma \mathrm{B}_{0}^{2} v}{\rho \mathrm{V}_{0}^{2}}, \mathrm{Sc}=\frac{v}{\mathrm{D}}, \mathrm{Sr}=\frac{\mathrm{D}_{\mathrm{T}}\left(\mathrm{T}_{\mathrm{w}}^{\prime}-\mathrm{T}_{\infty}^{\prime}\right)}{v\left(\mathrm{C}_{\mathrm{w}}^{\prime}-\mathrm{C}_{\infty}^{\prime}\right)} \\
& \mathrm{Kr}=\frac{v \gamma}{\mathrm{v}_{0}^{2}}, \mathrm{Gr}=\frac{v \beta \mathrm{g}\left(\mathrm{T}_{\omega}^{\prime}-\mathrm{T}_{\infty}^{\prime}\right)}{\mathrm{U}_{0} \mathrm{~V}_{0}^{2}}, \mathrm{Gm}_{0}=\frac{v \beta_{\mathrm{c}} \mathrm{g}\left(\mathrm{C}_{\mathrm{w}}^{\prime}-\mathrm{C}_{\infty}^{\prime}\right)}{\mathrm{U}_{0} \mathrm{~V}_{0}^{2}}
\end{aligned}
$$

From the equation (7)-(9), the governing equations (2)-(4) are reduced to the non- dimensional form as follows: $\frac{\partial \mathrm{u}}{\partial \mathrm{t}}-\frac{\partial \mathrm{u}}{\partial \mathrm{y}}=\frac{\partial \mathrm{U}}{\partial \mathrm{t}}+\frac{\partial^{2} \mathrm{u}}{\partial \mathrm{y}^{2}}+\mathrm{Gr} \theta+\mathrm{Gm} \phi+\mathrm{M}_{1}(\mathrm{U}-\mathrm{u})$

$\frac{\partial \theta}{\partial t}-\frac{\partial \theta}{\partial y}=\frac{1}{\operatorname{Pr}} \frac{\partial^{2} \theta}{\partial y^{2}}+N \theta$

$$
\frac{\partial \phi}{\partial \mathrm{t}}-\frac{\partial \phi}{\partial \mathrm{y}}=\frac{1}{\operatorname{Sc}} \frac{\partial^{2} \phi}{\partial \mathrm{y}^{2}}-\operatorname{Kr} \phi+\operatorname{Sr} \frac{\partial^{2} \theta}{\partial \mathrm{y}^{2}}
$$

Where

$$
\mathrm{M}_{1}=\mathrm{M}+\frac{1}{\mathrm{~K}}
$$

Applying non-dimensional variables given in equation (9), the boundary conditions (5) and (6) are reduced to the following dimensionless form as follows:

$$
\begin{aligned}
& \mathrm{u}=0, \quad \theta=1+\varepsilon \mathrm{e}^{\mathrm{i} \omega \mathrm{t}}, \quad \phi=1+\varepsilon \mathrm{e}^{\mathrm{i} \omega \mathrm{t}} \quad \text { at } \mathrm{y}=0 \\
& \mathrm{u} \rightarrow 1+\varepsilon \mathrm{e}^{\mathrm{i} \omega \mathrm{t}}, \quad \theta \rightarrow 0, \quad \phi \rightarrow 0 \quad \text { as } \mathrm{y} \rightarrow \infty
\end{aligned}
$$

$$
\mathrm{u}_{0} \quad 0, \mathrm{u}_{1} \quad 0, \quad 0 \quad 1, \quad \frac{1}{1} \quad 1
$$

\section{METHOD OF SOLUTION} Now we have needed to reduce the above sytstem'of partial 0
differential equations (10)-(12) into a system of ordinary differential equations in dimensionless form, we may consider the velocity, temperature and concentration as follows:

$$
u(y, t)=u_{0}(y)+\varepsilon e^{i \omega t} u_{1}(y)
$$

$\theta(\mathrm{y}, \mathrm{t})=\theta_{0}(\mathrm{y})+\varepsilon \mathrm{e}^{\mathrm{i} \omega \mathrm{t}} \theta_{1}(\mathrm{y})$

$$
\phi(\mathrm{y}, \mathrm{t})=\phi_{0}(\mathrm{y})+\varepsilon \mathrm{e}^{\mathrm{i} \omega \mathrm{t}} \phi_{1}(\mathrm{y})
$$

Where $\mathrm{u}_{0}, \theta_{0}, \phi_{0}$ are respectively the mean velocity, mean temperature and mean concentration.

Substituting the equations (14), (15) and (16) into the equation (10), (11) and (12), neglecting the higher of $\varepsilon$ and equating the harmonic and non-harmonic terms, we get following equations as follows:

$\left[\frac{\mathrm{d}^{2}}{\mathrm{dy}^{2}}+\frac{\mathrm{d}}{\mathrm{dy}}-\mathrm{M}_{1}\right] \mathrm{u}_{0}=-\mathrm{Gr} \theta_{0}-\mathrm{Gm} \phi_{0}-\mathrm{M}_{1}$

$$
\left[\frac{\mathrm{d}^{2}}{\mathrm{dy}^{2}}+\frac{\mathrm{d}}{\mathrm{dy}}-\left(\mathrm{M}_{1}+\mathrm{i} \omega\right)\right] \mathrm{u}_{1}
$$

$1=-\mathrm{Gr} \theta_{1}-\mathrm{Gm} \phi_{1}-\left(\mathrm{M}_{1}+\mathrm{i} \omega\right)$

$$
\left[\frac{\mathrm{d}^{2}}{\mathrm{dy}^{2}}+\operatorname{Pr} \frac{\mathrm{d}}{\mathrm{dy}}+\mathrm{NP}_{\mathrm{r}}\right] \theta_{0}=0
$$$$
\left[\frac{d^{2}}{d y^{2}}+\operatorname{Pr} \frac{d}{d y}+P_{r}(N-i \omega)\right] \theta_{1}=0
$$

$\left[\frac{\mathrm{d}^{2}}{\mathrm{dy}^{2}}+\mathrm{Sc} \frac{\mathrm{d}}{\mathrm{dy}}-\mathrm{KrSc}\right] \phi_{0}=-\operatorname{SrSc} \theta_{0}^{\prime \prime}$

$\left[\frac{d^{2}}{d y^{2}}+\operatorname{Sc} \frac{d}{d y}-\operatorname{Sc}(\operatorname{Kr}+\mathrm{i} \omega)\right] \phi_{1}=-\operatorname{SrSc} \theta_{1}^{\prime \prime}$

Applying the equations (14), (15), and (16) the boundary conditions (13) can be written as

$\mathrm{u}_{0}=0, \quad \mathrm{u}_{1}=0, \quad \theta_{0}=1, \theta_{1}=1, \quad 0 \quad 1, \quad 1 \quad 1, \quad$ at $\mathrm{y} \quad 0$

, $\phi_{0}=1, \phi_{1}=1, \quad$ at $\mathrm{y}=0$

$\mathrm{u}_{0}=1, \quad \mathrm{u}_{1}=1, \quad \theta_{0}=0, \quad 1 \quad 0, \quad 0 \quad 0, \quad 1 \quad 0, \quad$ at $\mathrm{y}$ $0, \theta_{1}=0, \phi_{0}=0, \phi_{1}=0, \quad$ at $\mathrm{y} \rightarrow \infty$

Solving the equations from (17) to (22) and applying the boundary conditions (23) and (24), getting following equations: 


$$
\begin{aligned}
& \theta_{0}(y)=e^{m_{1} y} \\
& \theta_{1}(y)=e^{m_{2} y} \\
& \phi_{0}(y)=\left(1-A_{1}\right) e^{m_{3} y}+A_{1} e^{m_{1} y} \\
& \phi_{1}(y)=\left(1-A_{2}\right) e^{m_{4} y}+A_{2} e^{m_{2} y} \\
& u_{0}(y)=\left(A_{3}+A_{4}+A_{5}-1\right) e^{m_{5} y} \\
& +1-A_{3} e^{m_{1} y}-A_{4} e^{m_{3} y}-A_{5} e^{m_{1} y} \\
& u_{1}(y)=A_{8} e^{m_{6} y}+1-A_{6} e^{m_{2} y}-A_{7} e^{m_{4} y}
\end{aligned}
$$

Where

$$
\begin{aligned}
& \mathrm{m}_{1}=\frac{-\operatorname{Pr}-\sqrt{\operatorname{Pr}^{2}-4 \mathrm{NPr}}}{2}, \\
& \mathrm{~m}_{2}=\frac{-\operatorname{Pr}-\sqrt{\operatorname{Pr}^{2}-4 \operatorname{Pr}(\mathrm{N}-\mathrm{i} \omega)}}{2} \\
& \mathrm{~m}_{3}=\frac{-\mathrm{Sc}-\sqrt{\mathrm{Sc}^{2}+4 \mathrm{KrSc}}}{2} \\
& \mathrm{~m}_{4}=\frac{\mathrm{A}_{\underline{8}} \mathrm{Sc}-\sqrt[\mathrm{A}_{6}]{\mathrm{Sc}^{\mathrm{A}^{2}}+{ }_{4} \mathrm{Sc}^{1}\left(\mathrm{Ar}_{9}+\mathrm{i} \omega\right)^{\mathrm{A}}}}{2}, \\
& M_{1}=M+\frac{1}{K}, \quad M=\frac{\sigma \beta_{o}^{2} v}{\rho v_{0}^{2}}, \quad K=\frac{K^{\prime} v_{o}^{2}}{v^{2}}, \\
& \mathrm{~m}_{5}=\frac{-1-\sqrt{1+4 \mathrm{M}_{1}}}{2} \\
& \mathrm{~m}_{6}=\frac{-1-\sqrt{1+4\left(\mathrm{M}_{1}+\mathrm{i} \omega\right)}}{2} \\
& \mathrm{~A}_{1}=\frac{-\mathrm{m}_{1}^{2} \mathrm{ScSr}}{\mathrm{m}_{1}^{2}+\mathrm{Scm}_{1}-\mathrm{KrSc}}, \\
& \mathrm{A}_{2}=\frac{-\mathrm{m}_{2}^{2} \mathrm{ScSr}}{\mathrm{m}_{2}^{2}+\mathrm{Scm}_{2}-\mathrm{Sc}(\mathrm{Kr}+\mathrm{i} \omega)} \\
& A_{3}=\frac{G r}{m_{1}^{2}+m_{1}-M_{1}}
\end{aligned}
$$

$$
A_{4}=\frac{G m\left(1-A_{1}\right)}{m_{3}^{2}+m_{3}-M_{1}}, \quad A_{5}=\frac{\mathrm{GmA}_{1}}{m_{1}^{2}+m_{1}-M_{1}}
$$$$
A_{6}=\frac{G r+G m A_{2}}{m_{2}^{2}+m_{2}-\left(M_{1}+i \omega\right)}
$$$$
A_{7}=\frac{G m\left(1-A_{2}\right)}{m_{4}^{2}+m_{4}-\left(M_{1}+i \omega\right)}, \quad A_{8}=A_{6}+A_{7}-1,
$$

From the equations (14) to (16), we obtain velocity, temperature and concentration as follows:

$$
\begin{aligned}
& u(y, t)=1+A_{9} e^{m_{5} y}-A_{10} e^{m_{1} y}-A_{4} e^{m_{3} y} \\
& +\varepsilon e^{i \omega t}\left[A_{8} e^{m_{6} y}+1-A_{6} e^{m_{2} y}-A_{7} e^{m_{4} y}\right] \\
& \theta(y, t)=e^{m_{1} y}+\varepsilon e^{i \omega t} e^{m_{2} y} \\
& \phi(y, t)=\left(1-A_{1}\right) e^{m_{3} y}+A_{1} e^{m_{1} y} \\
& +\varepsilon e^{i \omega t}\left[\left(1-A_{2}\right) e^{m_{4} y}+A_{2} e^{m_{2} y}\right]
\end{aligned}
$$

Where,

$A_{8}=A_{6}+A_{7}-1, A_{9}=A_{3}+A_{4}+A_{5}-1, A_{10} \quad A_{3} \quad A_{5}$ $A_{4} \quad A_{5} \quad 1, A_{10}=A_{3}+A_{5}$

Now we can calculate co-efficient of skin-friction at the wall of plate is given by

$$
\begin{aligned}
& \tau_{\omega}=\left[\frac{\partial \mathrm{u}}{\partial \mathrm{y}}\right]_{\mathrm{y}=0} \\
& =\mathrm{A}_{9} \mathrm{~m}_{5}-\mathrm{m}_{1} \mathrm{~A}_{10}-\mathrm{m}_{3} \mathrm{~A}_{4}+\varepsilon \mathrm{e}^{\mathrm{i} \omega \mathrm{t}}\left[\mathrm{A}_{8} \mathrm{~m}_{6}-\mathrm{A}_{6} \mathrm{~m}_{2}-\mathrm{A}_{7} \mathrm{~m}_{4}\right]
\end{aligned}
$$

The rate of heat transfer in terms of Nusselt number $\mathrm{Nu}$ ) as follows:

$\mathrm{Nu}=-\left[\frac{\partial \theta}{\partial \mathrm{y}}\right]_{\mathrm{y}=0}=-\mathrm{m}_{1}-\mathrm{m}_{2} \varepsilon \mathrm{e}^{\mathrm{i} \omega \mathrm{t}}$

The rate of mass transfer co-efficient in terms of Sherwood number as follows:

$$
\begin{aligned}
& \mathrm{Sh}=-\left[\frac{\partial \phi}{\partial \mathrm{y}}\right]_{\mathrm{y}=0} \\
& =-\left(1-\mathrm{A}_{1}\right) \mathrm{m}_{3}-\mathrm{m}_{1} \mathrm{~A}_{1}-\varepsilon \mathrm{e}^{\mathrm{i} \omega \mathrm{t}}\left[\left(1-\mathrm{A}_{2}\right) \mathrm{m}_{4}+\mathrm{A}_{2} \mathrm{~m}_{2}\right]
\end{aligned}
$$




\section{Thermal Diffusion (Soret Effect) on an Unsteady MHD}

\section{RESULTS AND DISCUSSIONS}

In the present discussion, the expressions of fluid velocity, temperature, concentration viscous drag, co-efficient of rate of heat transfer and co-efficient of rate of mass transfer are piled up to get a variety of graphs with their substantial interprtations by taking some random values of different parameters implicated in the problem.

The velocity profile under the influence of Hartmann number $\mathrm{M}$, Chemical reaction parameter Kr, Soret number $\mathrm{Sr}$ and Schmdit number Sc against the normal co-ordinate

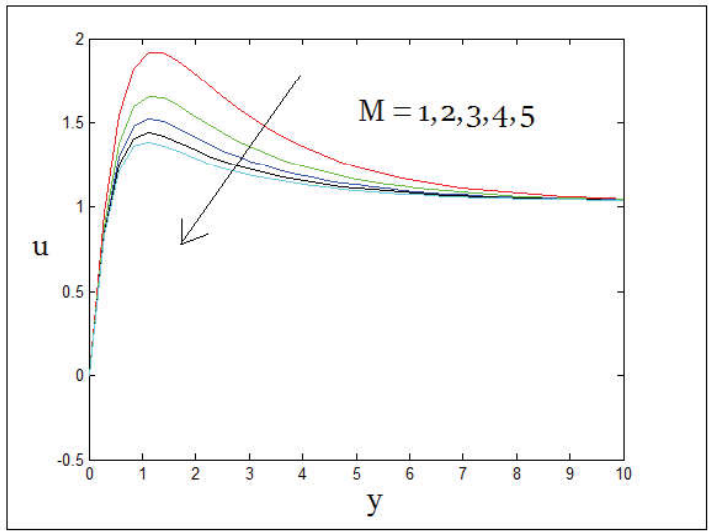

Fig. 2: Velocity Versus y under $\mathrm{K}=2$, $\mathrm{N}=0.2, \mathrm{Gr}=2, \mathrm{Gm}=2, \mathrm{Kr}=2, \mathrm{Sc}=0.22$, $\operatorname{Pr}=0.71, \mathrm{Sr}=2, \varepsilon=0.5, \omega=10, \mathrm{t}=0.15$

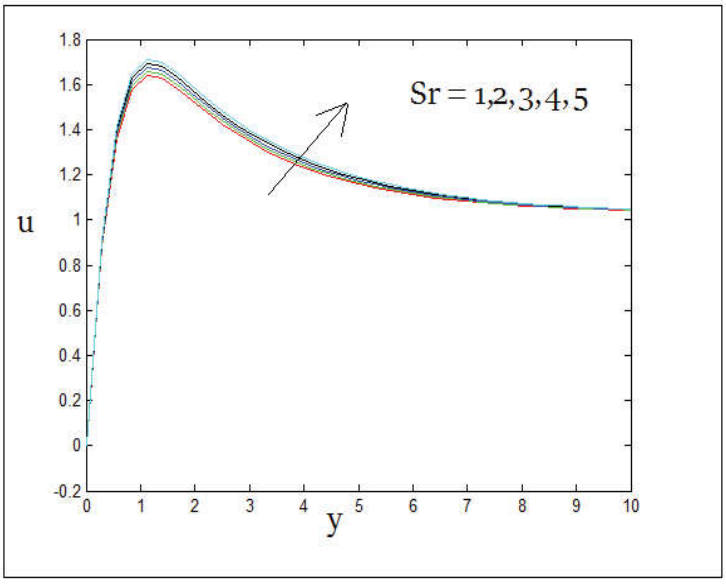

Fig. 4: Velocity Versus y under $\mathrm{K}=2$, $\mathrm{N}=0.2, \mathrm{Gr}=2, \mathrm{Gm}=2, \mathrm{Kr}=2, \mathrm{Sc}=0.22$, $\operatorname{Pr}=0.71, M=2, \varepsilon=0.5, \omega=10, t=0.15$ $\mathrm{y}$ are exhibited in figures 2-5. It is contingent from figure 2 and 3 that, an increase in magnetic parameter $M$ and chemical reaction parameter $\mathrm{Kr}$ has an inhibiting effect on fluid velocity. The fluid velocity is continuously condensed with increasing $\mathrm{M}$ and $\mathrm{Kr}$. In other words the obligation of the transverse magnetic field and reaction tends to slow down the fluid flow. In figure 5, we observe the same trend of performance with Schmdit number Sc is concerned. But figure 4 displays the opposite behavior with respect to the above figures. i.e. the fluid motion gets accelerated under the action of mass flux.

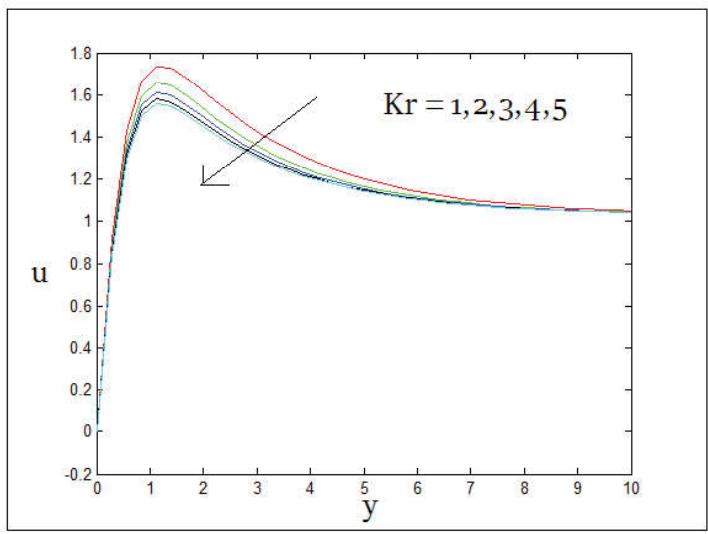

Fig. 3: Velocity Versus y under $\mathrm{K}=2$, $\mathrm{N}=0.2, \mathrm{Gr}=2, \mathrm{Gm}=2, \mathrm{M}=2, \mathrm{Sc}=0.22$, $\operatorname{Pr}=0.71, \mathrm{Sr}=2, \varepsilon=0.5, \omega=10, \mathrm{t}=0.15$

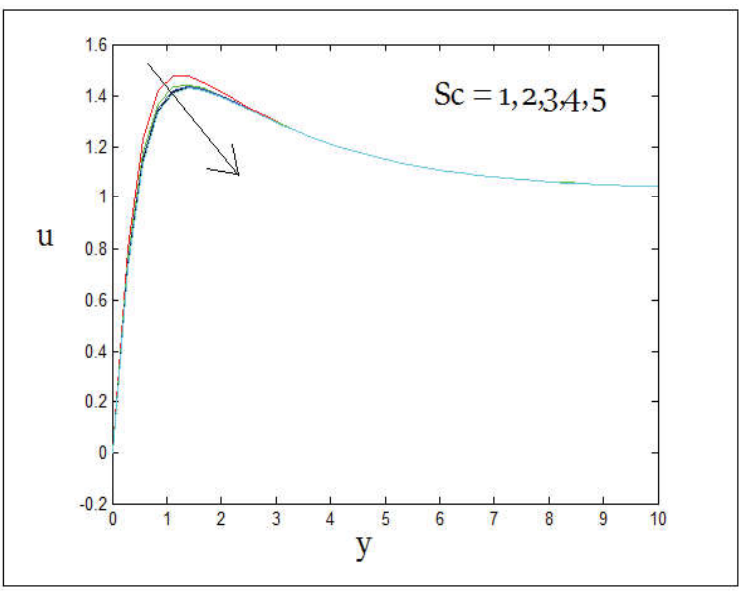

Fig. 5: Velocity Versus y under $\mathrm{K}=2$, $\mathrm{N}=0.2, \mathrm{Gr}=2, \mathrm{Gm}=2, \mathrm{Kr}=2, \mathrm{M}=2$, $\operatorname{Pr}=0.71, \mathrm{Sr}=2, \varepsilon=0.5, \omega=10, \mathrm{t}=0.15$; 


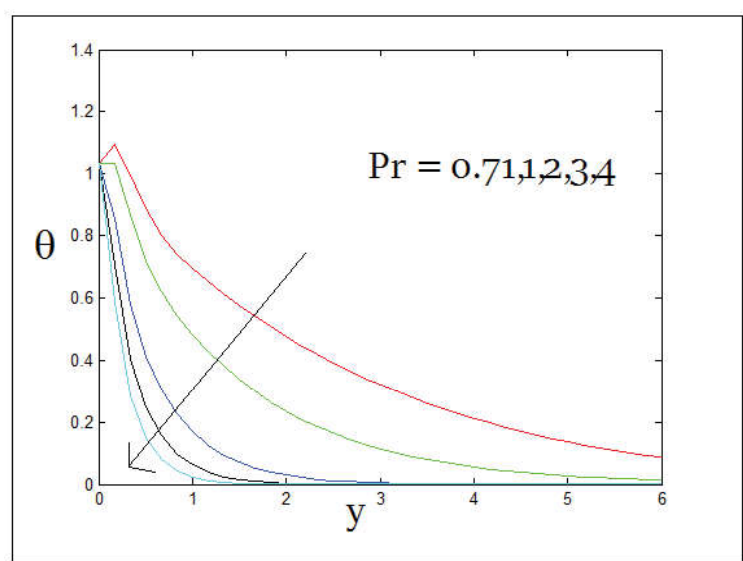

Fig. 6: Temperature Versus y under $\mathrm{N}=0.2, \varepsilon=0.5, \omega=10, \mathrm{t}=0.1$

Figures 6 to 7 match up to the temperature distribution $\theta$ against $y$ under the effects of the Prandtl number $\operatorname{Pr}$ and radiation parameter $\mathrm{N}$. Rising of $\mathrm{Pr}$ or $\mathrm{N}$ shows a contrasting control on $\theta$ which indicates the fact that the fluid temperature falls down gradually for increasing values of Pr and R. It is further noticed from these figures that the fluid temperature as expected asymptotically falls from its maximum value at $\mathrm{y}=0$ to its minimum value at $\mathrm{y} \rightarrow \infty$.

The effects of Soret number Sr and Schmdit number Sc on species concentration have been displayed in figures 8 and 9. It is contingent from both the figures that concentration

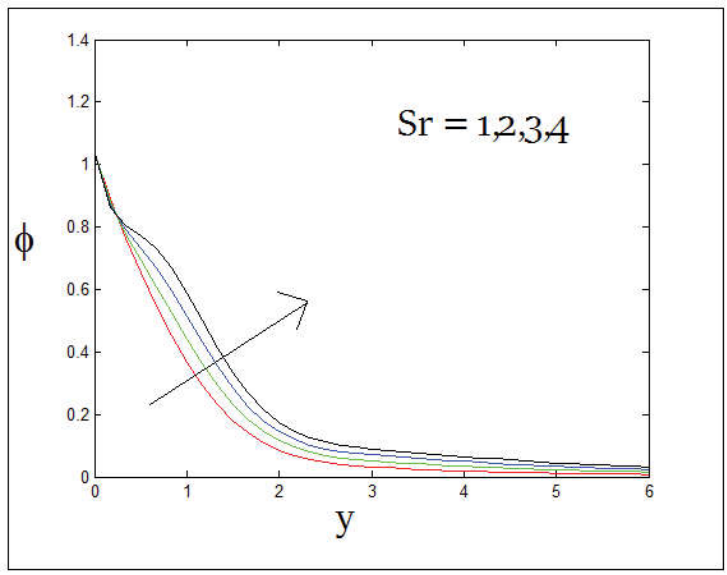

Fig. 8: Concentration Versus y under $\mathrm{N}=0.2$, $\varepsilon=0.5, \omega=10, \mathrm{t}=0.15, \mathrm{Pr}=0.71, \mathrm{Sc}=0.60, \mathrm{Kr}=2$

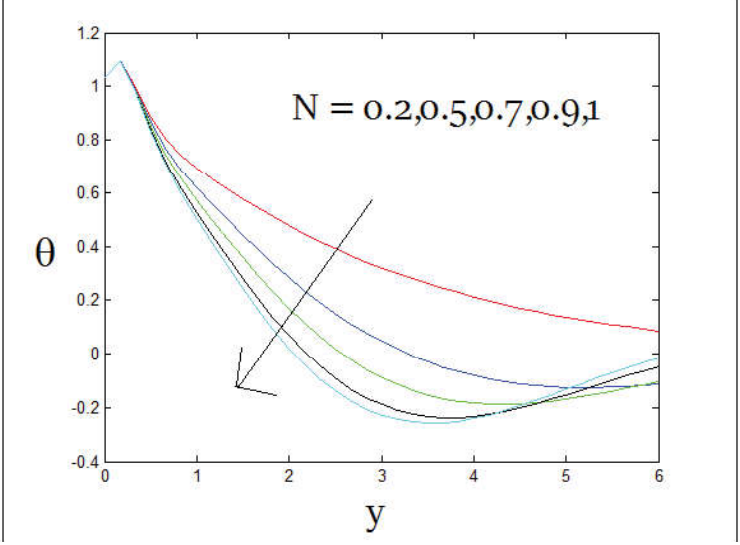

Fig. 7: Temperature Versus y under $\operatorname{Pr}=0.71, \varepsilon=0.5, \omega=10, t=0.15$

level of the fluid quickly rises up for increasing values of Soret number Sr and drops for increasing Schmdit number Sc. It indicates that the concentration level gets enhanced due to mass flux and high mass diffusivity.

The variations of skin friction against $\omega$ under the influence of Hartmann number $M$, Chemical reaction parameter $\mathrm{Kr}$, Soret number $\mathrm{Sr}$ and Schmdit number Sc are presented in figures 10-13. It is observed from the figures 10, 11 and 13 that an increase in magnetic intensity, chemical reaction and mass diffusivity tends to minimize the viscous drag. It is seen from figure 12 that the friction is raised by virtue of thermal diffusion effect.

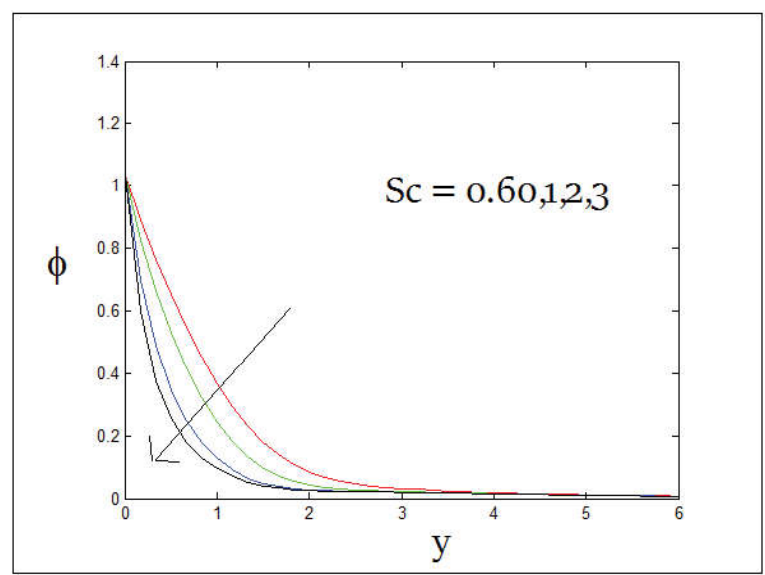

Fig. 9: Concentration Versus y under $\mathrm{N}=0.2$, $\varepsilon=0.5, \omega=10, \mathrm{t}=0.15, \mathrm{Pr}=0.71, \mathrm{Sr}=1, \mathrm{Kr}=2$ 


\section{Thermal Diffusion (Soret Effect) on an Unsteady MHD}

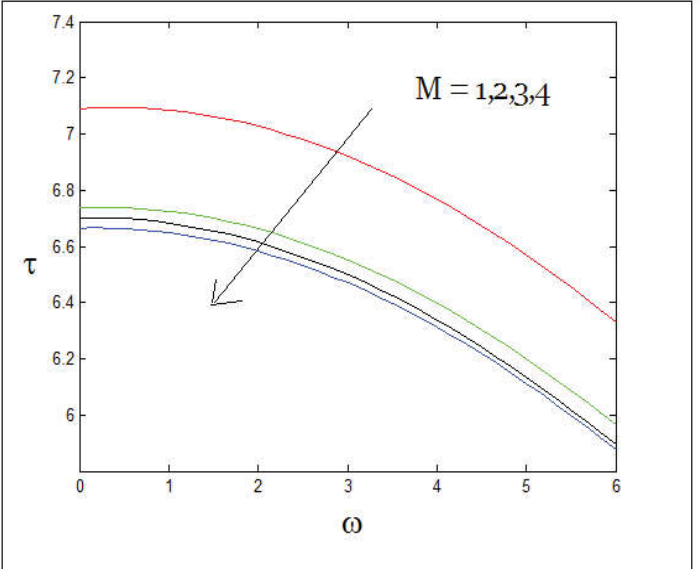

Fig. 10: Skin Friction Versus $\omega$ under $K=2, N=0.2, G r=2$, $\mathrm{Gm}=2, \mathrm{Kr}=2, \mathrm{Sc}=0.22, \operatorname{Pr}=0.71, \mathrm{Sr}=2, \varepsilon=0.5, \mathrm{t}=0.15$

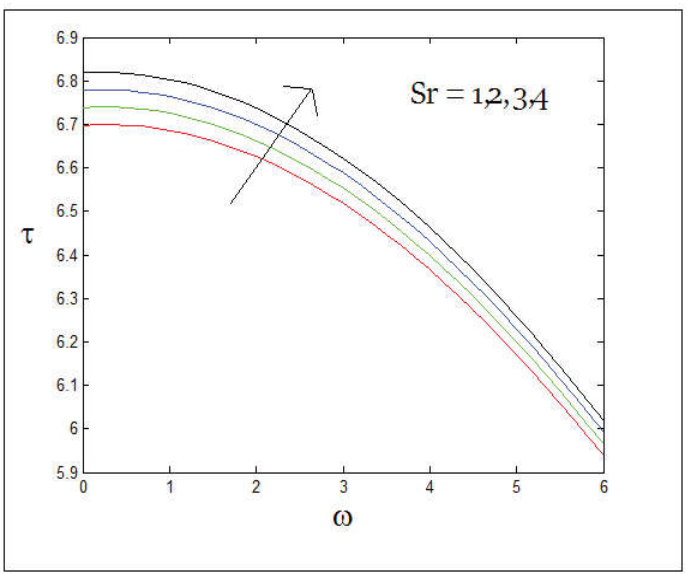

Fig. 12: Skin Friction Versus $\omega$ under $K=2, N=0.2, G r=2$, $\mathrm{Gm}=2, \mathrm{Kr}=2, \mathrm{Sc}=0.22, \mathrm{M}=2, \mathrm{t}=0.15, \operatorname{Pr}=0.71, \varepsilon=0.5$

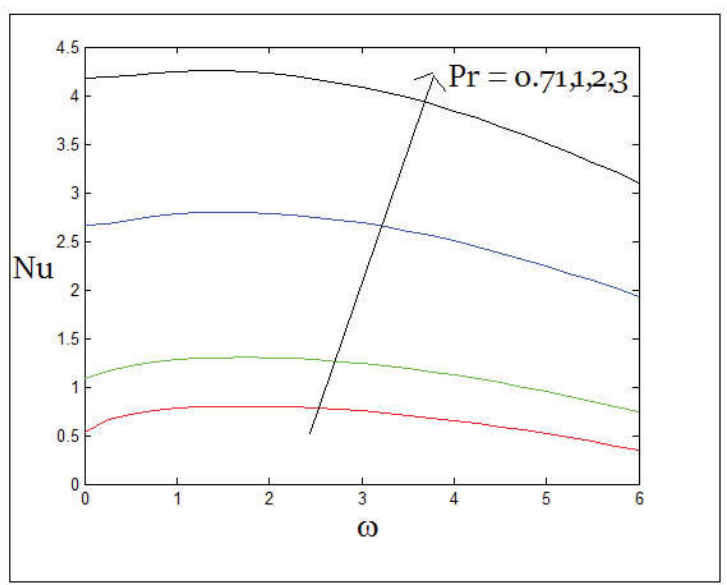

Fig. 14: Nusselt Versus $\omega$ under $\mathrm{N}=0.2$, $\varepsilon=0.5, \mathrm{t}=0.15$

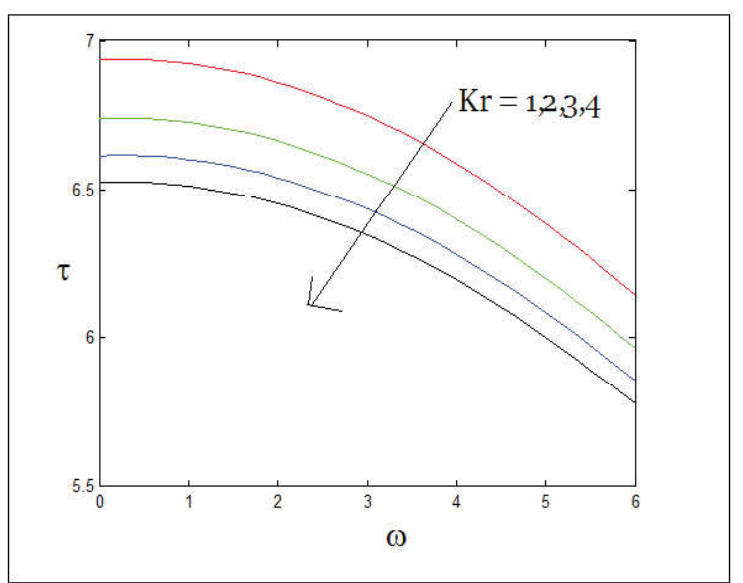

Fig. 11: Skin friction versus $\omega$ under $K=2, N=0.2, G r=2$, $\mathrm{Gm}=2, \mathrm{M}=2, \mathrm{Sc}=0.22, \mathrm{Pr}=0.71, \mathrm{Sr}=2, \varepsilon=0.5, \mathrm{t}=0.15$

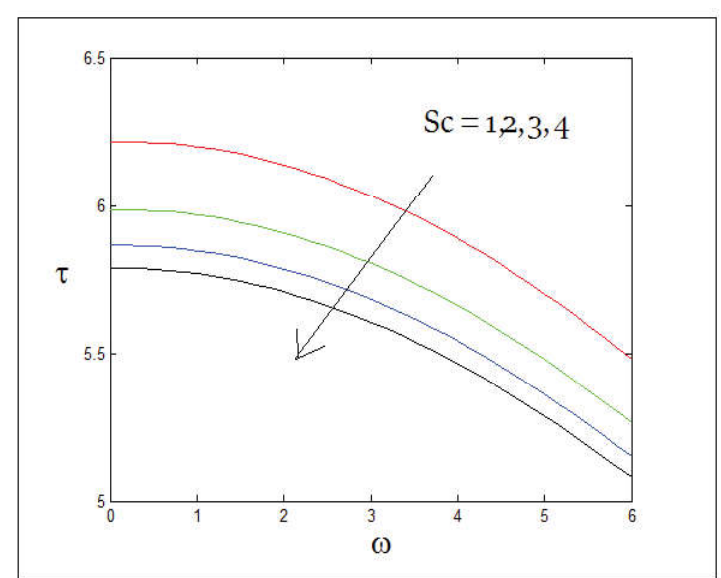

Fig. 13: Skin Friction Versus $\omega$ under $\mathrm{K}=2, \mathrm{~N}=0.2, \mathrm{Gr}=2$, $\mathrm{Gm}=2, \mathrm{M}=2, \mathrm{Kr}=2, \mathrm{Pr}=0.71, \mathrm{Sr}=2, \varepsilon=0.5, \mathrm{t}=0.15$

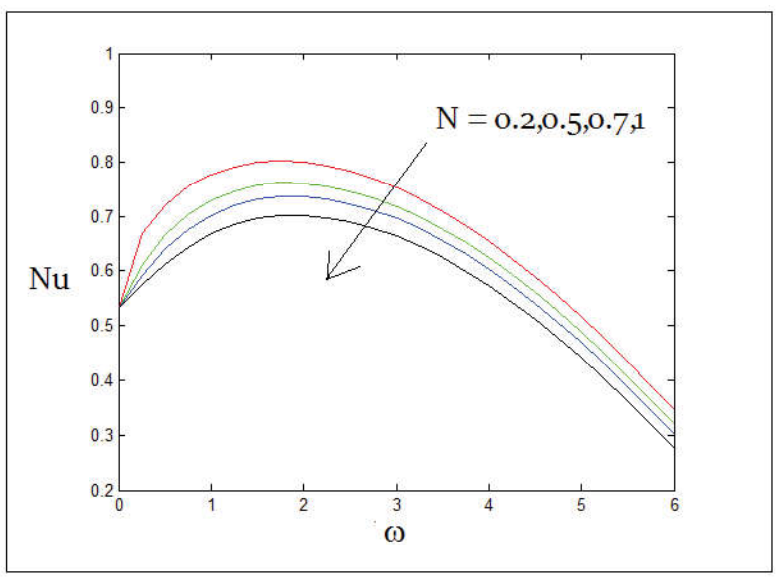

Fig. 15: Nusselt Versus $\omega$ under $\operatorname{Pr}=0.71$, $\varepsilon=0.5, t=0.15$ 


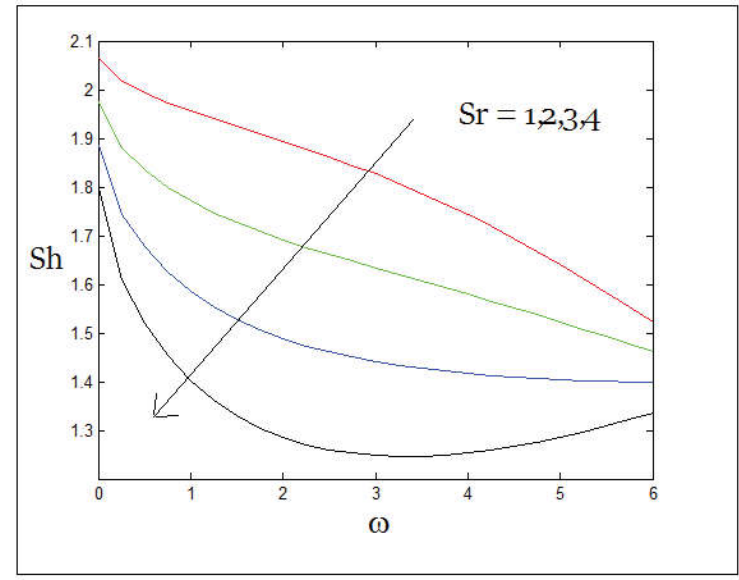

Fig. 16: Sherwood Versus $\omega$ under $N=0.2$ $\varepsilon=0.5, \mathrm{t}=0.15, \mathrm{Pr}=0.71, \mathrm{Sc}=0.60, \mathrm{Kr}=2$

The effects of Prandtl number $\mathrm{Pr}$ and radiation parameter $\mathrm{N}$ on the co-efficient of rate of heat transfer in terms of Nusselt number have been displayed in figures $14-15$. It is found from figure 14 that the magnitude of the rate of heat transfer increases with the increase in Prandtl number Pr. This simulates that low thermal diffusivity leads the substantial rise in the heat transfer rate. Figure 15 demonstrates that the co-efficient of rate of heat transfer is reduced with the increasing values of radiation parameter $\mathrm{N}$. ie the energy flux falls due to low thermal conductivity.

The figures 16 and 17 present the variation of the rate of mass transfer from the plate to the fluid. It is inferred from figure 16 that the Sherwood number falls down under the action of thermal diffusion effect. It is contingent from figure 17 that the co-efficient of rate of mass transfer rises under Schmdit number. i.e. the mass flux from the plate to the fluid get increased under the influence of mass diffusivity.

\section{CONCLUSION}

The following conclusions can be drawn from the present investigation of the problem:

1. The primary fluid motion is retarded under the action of transverse magnetic field and accelerated in presence of Soret number.

2. The concentration level of the fluid quickly rises up for increasing values of Soret number $\mathrm{Sr}$ and drops for increasing Schmdit number Sc. It indicates that the concentration level gets enhanced due to mass flux and high mass diffusivity.

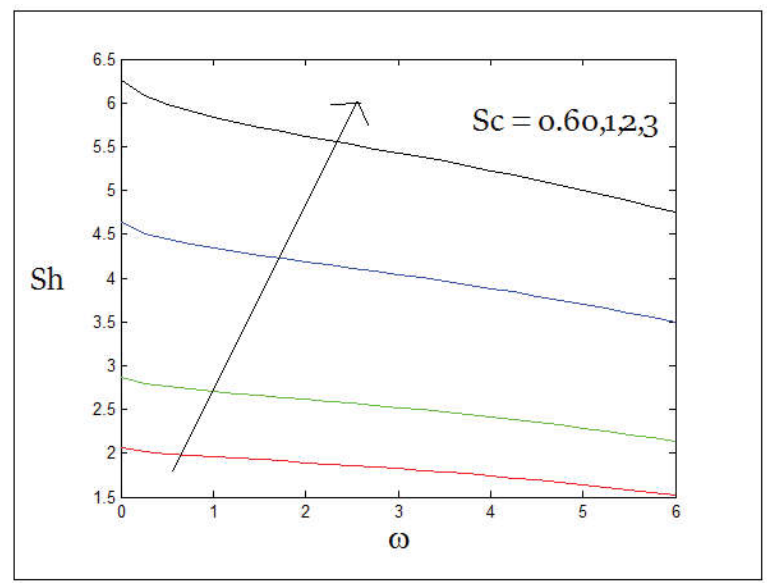

Fig. 17: Sherwood Versus $\omega$ under $N=0.2$, $\operatorname{Pr}=0.71, \mathrm{Sr}=1, \mathrm{Kr}=2, \varepsilon=0.5, \mathrm{t}=0.15$

3. An increase in magnetic intensity and chemical reaction tends to minimize the viscous drag and the friction is raised by virtue of thermal diffusion effect.

4. Sherwood number falls down under the action of thermal diffusion effect and the co-efficient of rate of mass transfer rises under Schmdit number. i.e. the mass flux from the plate to the fluid get increased under the influence of mass diffusivity.

\section{NOMENCLATURE}

U Non dimension velocity along the plate

$\mathrm{U}_{0} \quad$ Mean stream velocity

U' Dimensional velocity along the plate

$\mathrm{x}, \mathrm{y}$ distances along and perpendicular to the plate, respectively

$\mathrm{V}_{\mathrm{o}} \quad$ Constant suction velocity

$\mathrm{x}^{\prime}, \mathrm{y}^{\prime}$ dimensionless distances along and perpendicular to the plate, respectively

$\mathrm{u}, \mathrm{v} \quad$ components of dimensionless velocities along $\mathrm{x}^{\prime}$ and $\mathrm{y}^{\prime}$ directions respectively

$\mathrm{u}^{\prime}, \mathrm{v}^{\prime}$ components of dimensional velocities along $\mathrm{x}^{\prime}$ and $\mathrm{y}^{\prime}$ directions respectively

$\mathrm{t}$ non-dimensional time

$\mathrm{t}^{\prime}$ dimensional time

D Diffusion co-efficient 
$\mathrm{D}_{\mathrm{T}} \quad$ Thermal Diffusion Ratio

$\mathrm{K}_{\mathrm{r}} \quad$ Non-dimension chemical reaction parameter

$\mathrm{K}_{\mathrm{r}}^{\prime} \quad$ Dimensional chemical reaction parameter

K Non-dimension porosity number

$\mathrm{K}^{\prime} \quad$ Dimensional porosity number

$\mathrm{T}^{\prime} \quad$ dimensional temperature of the fluid

$\mathrm{T}_{\mathrm{w}}^{\prime} \quad$ dimensional temperature at the plate

$\mathrm{T}_{\infty}^{\prime} \quad$ dimensional temperature in the free stream

N Radiation parameter

K non-dimension porosity parameter

$\mathrm{P}_{\mathrm{r}} \quad$ Prandtl number

M Magnetic parameter

$\mathrm{Gr}$ Grashoff number

$\mathrm{G}_{\mathrm{m}} \quad$ Grashoff number for mss transfer

$\mathrm{S}_{\mathrm{c}} \quad$ Schmdit number

$\mathrm{Sr} \quad$ Soret number

$C^{\prime}$ dimensional concentration

$\mathrm{C}_{\mathrm{w}}^{\prime} \quad$ dimensional concentration at the wall

$\mathrm{C}_{\infty}^{\prime}$ dimensional temperature in the free stream

g Acceleration due to gravity

$\mathrm{p} \quad$ Non dimension pressure

$\mathrm{p}^{\prime} \quad$ Dimensional pressure

$\mathrm{C}_{\mathrm{p}} \quad$ Specific heat at constant pressure

\section{GREEK SYMBOLS}

$\rho \quad$ Density of the fluid

$v \quad$ fluid kinetic viscosity

$\alpha \quad$ Radiation absorption co-efficient

$\beta \quad$ co-efficient of thermal expansion

$\beta_{c} \quad$ Volumetric expansion co-efficient

$\theta$ non-dimensional temperature

$\sigma \quad$ Electrical conductivity

$\phi$ non-dimensional concentration

$\varepsilon \quad$ small reference parameter

$\omega \quad$ Frequency parameter

\section{SUBSCRIPTS}

w Wall condition

$\infty \quad$ Free stream condition

\section{SUPERSCRIPTS}

/ Dimensional properties

// Differentiation w. r. to y

\section{REFERENCES}

Ahmed N (2010) MHD convection with Soret and Dufour effects in a three dimensional flow past an infinite vertical porous plate. Can. Jr. Phys. 88(9): 663-674.

Ahmed N (2010) MHD free and forced convection with mass transfer from an infinite vertical porous plate. Journal of Energy, Heat and Mass Transfer 32: 55-70.

Alam S, Rahman M M, Maleque A and Ferdows M (2004) Dufour and Soret effects on steady MHD combined free-forced convective and mass transfer flow past a semi-infinite vertical plate. Thammasat Int. J. Sc. Tech. 11(2): 1-12.

Allan M M and Dardery S M (2018) On the effect of convective heat and mass transfer on unsteady mixed convection MHD flow through vertical porous medium. International Journal of Physical Sciences 13.5: 66-78.

Anderson H I, Hansen O R and Holmedal B (1994) Diffusion of a chemically reactive species from a stretching sheet. International Journal of Heat and Mass Transfer 37: 659-664.

Anghel M, Takhar H S and Pop I (2000) Dufour and Soret effects on free convection boundary layer over a vertical surface embedded in porous medium. Studiab Universitatis BabesBolyai Mathematica 60(5): 11-21.

Elbashbeshy E M A (2003) The mixed convection along a vertical plate embedded in non- darcian porous medium with suction and injection Applied Mathematics and Computation 136(1): 139-149.

Muthukumaraswami R (2002) Effects of a chemical reaction on a moving isothermal surface with suction. Acta Mechanica 155: 65-72.

Postelnicu A (2004) Influence of a magnetic field on Heat and Mass Transfer by natural convection from a vertical surface in porous media considering Soret and Dufour effects. Int. J. of Heat and Mass Transfer 47: 1467-1472.

Singh N P and Singh Atul K (2000) MHD effects on heat and mass transfer in a flow of viscous fluid with induced magnetic field. Indian Journal of Pure and Applied Physics 38: 182-189.

Apelblat A (1982) Mass transfer with a chemical reaction of the first order effect of axial diffusion. The chemical Engineering Journal 23: 193-203.

Chambre P L and Young J D (1958) On the diffusion of a chemically reactive species in a laminar boundary layer flow. Phys. Fluids Flow 1: 48-54. 